Provided for non-commercial research and education use. Not for reproduction, distribution or commercial use.

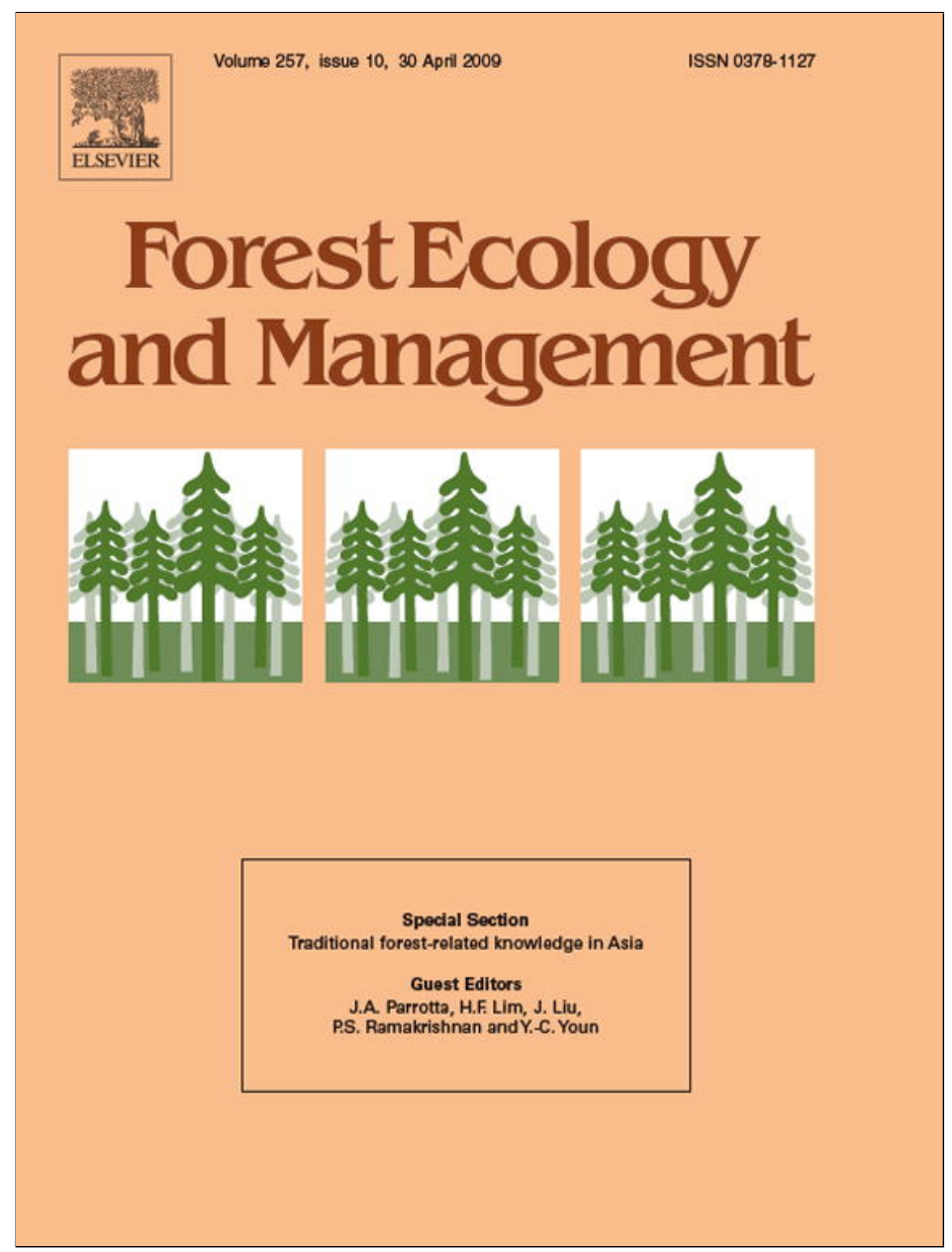

This article appeared in a journal published by Elsevier. The attached copy is furnished to the author for internal non-commercial research and education use, including for instruction at the authors institution and sharing with colleagues.

Other uses, including reproduction and distribution, or selling or licensing copies, or posting to personal, institutional or third party websites are prohibited.

In most cases authors are permitted to post their version of the article (e.g. in Word or Tex form) to their personal website or institutional repository. Authors requiring further information regarding Elsevier's archiving and manuscript policies are encouraged to visit:

http://www.elsevier.com/copyright 


\title{
Estimating the volume of forest growing stock using auxiliary information derived from relascope or ocular assessments
}

\author{
Piermaria Corona ${ }^{\mathrm{a}, *}$, Lorenzo Fattorini ${ }^{\mathrm{b}}$, Sara Franceschi ${ }^{\mathrm{b}}$

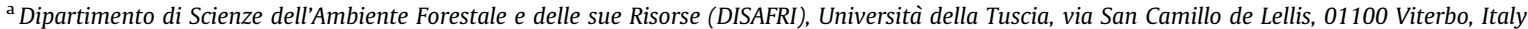 \\ ${ }^{\mathrm{b}}$ Dipartimento di Metodi Quantitativi (DMQ), Università di Siena, P.za S. Francesco 8, 53100 Siena, Italy
}

\section{A R T I C L E I N F O}

\section{Article history:}

Received 21 October 2008

Received in revised form 12 February 2009

Accepted 12 February 2009

\section{Keywords:}

Forest inventory by compartments

Two-phase sampling

Double-expansion estimators

Ratio estimators

Monte Carlo studies

\begin{abstract}
A B S T R A C T
The aim of this study is to demonstrate the potential of integrating probabilistic sampling and estimation with the conventional technique referred to as forest inventory by compartments. The objective of this paper is to propose two strategies for the assessment of growing stock volume using two-phase sampling, namely: (i) relascope basal area estimation performed on first-phase sampling points followed by volume estimation performed on a sub-sample of points selected in the second phase; (ii) ocular evaluation of growing stock volume performed on first-phase sampling plots of fixed size followed by volume estimation performed on a sub-sample of plots selected in the second phase. The effectiveness of using the auxiliary information gathered in the first phase is assessed by comparing the doubleexpansion estimator of total volume which depends solely on the second-phase sample with the twophase ratio estimator which instead calibrates the double-expansion estimator on the basis of firstphase information. Conservative estimators of sampling variances and confidence intervals are derived for both the estimators. As is usual in forest inventories, first-phase sampling is assumed to be performed on a systematic random grid while three different schemes are considered for drawing the second-phase sample: simple random sampling without replacement, stratified sampling and 3-P sampling. The performance of double-expansion and ratio estimators under the three schemes adopted in the second phase is empirically checked by means of a simulation study performed on a real compartment in a beech forest of Central Italy. Simulation results show that the use of auxiliary information generated in the first phase constitutes a very effective way of increasing the accuracy of volume estimation at the compartment level, with a moderate increase of fieldwork.
\end{abstract}

(c) 2009 Elsevier B.V. All rights reserved.

\section{Introduction}

Information about the state of and changes to forest stands is fundamental for environmental and timber assessment at various levels of forest management planning (Schreuder et al., 1993; Köhl et al., 2006). Data acquisition is usually based on standwise field inventories which provide updated figures for each forest management unit. Such a survey approach is commonly referred to as forest inventory by compartments (Koivuniemi and Korhonen, 2006). In European countries compartment means a relatively homogeneous forest area usually about $1-30$ ha in size.

Growing stock volume is a key attribute of inventory by compartments. All compartments need to be visited in the field, and the typical problem is the disparity between the required amount of fieldwork and available resources. In countries characterized by high labour costs, the assessment of growing

\footnotetext{
* Corresponding author. Tel.: +39 0761 357425; fax: +39 0761357389.

E-mail address: piermaria.corona@unitus.it (P. Corona).
}

stock volume at compartment level may be so expensive as to jeopardize management planning.

A solution to this problem has traditionally been found in terms of subjective methods exploiting relascope or ocular assessments (Koivuniemi and Korhonen, 2006). For many decades, these methods have widely replaced those based on full-tree callipering over large tracts, which however still takes place (e.g., in some areas of Alpine and Eastern European countries).

Despite their popularity, subjective inventory methods preclude rigorous evaluations about the uncertainty of estimates and, according to various Authors (e.g., Laasasenaho and Paivinen, 1986; Nersten and Næsset, 1992; Stähl, 1992; Kinnunen et al., 2007), they are also unreliable. Indeed, as observed by Koivuniemi and Korhonen (2006), even a seemingly highly homogeneous compartment may exhibit considerable variation in growing stock volumes. Accordingly, the forest technician's impression of the average amount of growing stock along a walked route may greatly differ from the actual volume, especially when, as usual, technicians do not visit each part of a compartment. In Scandinavian forests, the accuracy of stand-level inventories for basal area may vary from 13 
to $22 \%$ and bias can reach $10 \%$ (Haara, 2005), while the accuracy of stand volume estimates may vary from 15 to $45 \%$ (Haara, 2005) and the bias may exceed 20\% (Nersten and Næsset, 1992; Stähl, 1992).

Over the last decades, various technological advancements have opened up new opportunities in forest inventories. In particular, the field positioning of sampling units has been greatly facilitated due to the advances and practical availability of global positioning systems. This fact has enhanced the practical suitability of performing inventories via probabilistic procedures based on systematic or random positioning of the sampling units.

The aim of this study is to demonstrate the potential of integrating probabilistic sampling and estimation with conventional techniques of forest inventory by compartments. Two strategies for the estimation of growing stock volume by twophase sampling are proposed and tested in a complete designbased framework (Gregoire, 1998):

i. relascope basal area estimation performed on first-phase sampling points followed by volume estimation performed on a sub-sample of points selected in the second phase;

ii. ocular evaluation of growing stock volume performed on firstphase sampling plots of fixed size followed by volume estimation performed on a sub-sample of plots selected in the second phase.

The paper deals with the theoretical aspects and practical implementation of these two strategies. In Section 2, doubleexpansion and ratio estimators of total volume at compartment level are considered when the first-phase sampling is performed on a systematic random grid; related sampling variance estimators and confidence intervals are also proposed. For the second-phase sampling, three different schemes are considered: simple random sampling without replacement, stratified sampling and sampling with probability proportional to prediction (usually referred to as 3-P sampling). In Section 3, the performance of double-expansion and ratio estimators is empirically checked for each of the secondphase sampling schemes by means of a simulation study carried out on a real population. Finally, in Section 4, we frame the results in our experience in the perspective of practical applications.

\section{Methods}

\subsection{First-phase sampling}

Let $\mathrm{U}$ be a population of trees on a delineated study area $\mathcal{A}$ (i.e., a forest compartment) of size $A$. Denote by $y_{j}$ the volume of the $j$ th tree, where in accordance with FAO (2004) volume refers to the over bark part of living trees from the stump to the top of the bole, including main branches (see FAO, 2004). It is worth noting that actual volumes are prohibitive to measure and they are usually quantified by means of some standard dendrometric equations predicting the volume as a function of stem diameter at breast height and tree height (van Laar and Akça, 2007). Accordingly, henceforth $y_{j}$ will denote the predicted volume of the $j$ th tree, rather than its actual value. Now, suppose the total (predicted) volume

$T P V=\sum_{j \in U} y_{j}$

is the quantity to be estimated by means of an inventory involving $N$ points or plots selected over the study area.

The basic reference sampling scheme to select $N$ points on a study area is random sampling in which points are randomly and independently thrown over the area. Even if this scheme is commonly suggested and gives rise to straightforward statistical analysis, it is likely to produce unsuitable voids (i.e., undetected parts) in the study area. Therefore, in order to ensure a systematic search over the area, a stratified scheme is usually preferred by foresters. One such scheme involves covering the area by means of $N$ rectangles, quadrats or other regular polygons of the same size, each of them containing at least a portion of the study area, and then selecting a point in each of these polygons. If the points are randomly and independently selected within each polygon, the scheme is usually referred to as unaligned systematic sampling (see e.g., the EPA, 2002, p. 63), while if a point is randomly selected within one polygon and the other points are selected in the same relative positions in the remaining polygons, the scheme is referred to as aligned systematic sampling (see e.g., the EPA, 2002, p. 70).

Barabesi (2003) has theoretically proven that the unaligned systematic scheme provides estimators with variances invariably smaller than those achieved under random sampling, while this cannot be proven for the aligned scheme. Indeed, in the presence of some spatial regularity, the variances arising from the aligned scheme may be even greater than those provided by the random scheme. However, apart from certain situations in which spatial regularities are present, the performance of the aligned scheme is very similar (sometimes superior) to that of the unaligned scheme. The aligned scheme is often adopted by forest technicians since it can be executed by a random shift of a grid superimposed onto a map of the study area, taking the nodes as sample points and locating the points in the terrain by a modern-day GPS system.

On the basis of these considerations, suppose that before starting the survey the study area is covered by a region, say $R \supset A$, of size $R$ and constituted by $N$ non-overlapping quadrats $Q_{1}, Q_{2}, \ldots, Q_{N}$ of equal size and such that $Q_{i} \cap A \neq \varnothing$ for all $i=1$, $2, \ldots, N$. Usually, the size of the quadrats is chosen by forest technicians on the basis of practical considerations, while the grid is constructed by using arbitrary orientation and an arbitrary starting point. After the aligned systematic sampling has been performed, a collection of $N$ first-phase points, say $\mathrm{P}=\left\{\mathbf{u}_{1}, \ldots, \mathbf{u}_{N}\right\}$, is obtained. Subsequently, Bitterlich sampling or plot sampling are presumed to be carried out in each of these points. It is worth noting that, owing to the costs and time involved, in real situations Bitterlich or plot sampling cannot be performed for each firstphase point, but rather for a portion of these points selected in the second phase. Accordingly, the first-phase survey is only hypothetical and its treatment has the sole aim of constructing the theoretical basis for the subsequent analysis of the second phase.

In the case of Bitterlich sampling, for each first-phase point $\mathbf{u}_{i}$, all the trees subtending an angle less than a prefixed angle $\theta$ onto the point are selected (see e.g., Gregoire and Valentine, 2008, chapter 8). Then, if $S_{i}$ denotes the set of trees sampled at point $\mathbf{u}_{i}$, the HorvitzThompson estimator of TPV at this points turns out to be

$Y_{i}=R \sin ^{2} \frac{\theta}{2} \sum_{j \in S_{i}} \frac{y_{j}}{b_{j}} \quad, i=1, \ldots, N$

where $b_{j}$ denotes the basal area of the tree, expressed in the same unit adopted for $R$. When, as usual, $R$ is expressed in hectares and the $b_{j} \mathrm{~s}$ in $\mathrm{m}^{2}$, then the constant $10^{4} \sin ^{2}(\alpha / 2)$ is named Basal Area Factor (BAF) and is most frequently specified as the characteristic of the measurement device instead of the opening angle $\theta$.

Alternatively, in the case of plot sampling, a circular plot of a pre-fixed size $a$ is centred at each point $\mathbf{u}_{i}$, in such a way that $S_{i}$ is constituted by all the trees lying within the circle (see e.g., Gregoire and Valentine, 2008, chapter 7). In this case, the Horvitz-Thompson estimator of TPV at $\mathbf{u}_{i}$ turns out to be

$Y_{i}=\frac{R}{a} \sum_{j \in S_{i}} y_{j}, \quad i=1, \ldots, N$

It is worth noting that some edge effects may be present owing to those trees positioned near the internal edge of the study region, which will have inclusion probabilities smaller than $b_{j} /\left\{R \sin ^{2}(\theta / 2)\right\}$ or $a / R$. A long list of correction methods has been 
proposed in order to avoid the negative bias induced by these edge effects (see e.g., Gregoire and Valentine, 2008, Section 7.5). Fortunately, in this framework, the aligned scheme performs like the correction method usually referred to as the buffer method (e.g., Gregoire and Valentine, 2008, Section 7.5.1), which entails allowing sample points to fall outside the boundary of $A$, but within some larger region that includes $A$. Under the aligned scheme the presence of units whose inclusion zone overlaps the boundary of the enlarged region $R \supset A$ should become negligible. Thus, edge effects can be ignored throughout the paper with no detrimental effect on the bias of the estimator (see also Section 3.4).

Owing to the aligned systematic selection of points, $Y_{1}, \ldots, Y_{N}$ do not constitute independent realizations of the Horvitz-Thompson estimator. Rather, they are realizations of $N$ correlated random variables, having different expectations $E_{\mathrm{P}}\left(Y_{i}\right)$, different variances $V_{\mathrm{P}}\left(Y_{i}\right)$ and covariances $C_{\mathrm{P}}\left(Y_{i}, Y_{h}\right)$ for any $h>i=1, \ldots, N$. In this framework, the operators $E_{\mathrm{P}}(\cdot), V_{\mathrm{P}}(\cdot)$ and $C_{\mathrm{P}}(\cdot)$ refer to all the possible sets of points $P$ which can be selected in the first phase by means of aligned systematic sampling. While expectations, variances and covariances of the $Y_{i}$ s have cumbersome analytical formulations, by using some analogies with Monte Carlo integration, Barabesi (2003) has shown that their arithmetic mean

$\bar{Y}=\frac{1}{N} \sum_{i=1}^{N} Y_{j}$

constitutes an unbiased estimator of $T P V$ with variance

$V_{P}(\bar{Y})=\frac{1}{N^{2}} \sum_{i=1}^{N} V_{P}\left(Y_{i}\right)+\frac{2}{N^{2}} \sum_{h>i=1}^{N} C_{P}\left(Y_{i}, Y_{h}\right)$

As previously pointed out, nothing ensures that (2) is smaller than the corresponding variance obtained under random sampling. Moreover, since only one point is selected within each quadrat, there is no way to achieve unbiased estimators of (2). This is a common problem with stratified spatial sampling when only one point is selected per stratum. Under the unaligned scheme, owing to the independence among the $Y_{i}$ s, their variance divided by $N$, say

$\frac{S_{Y}^{2}}{N}=\frac{1}{N(N-1)} \sum_{i=1}^{N}\left(Y_{i}-\bar{Y}\right)^{2}$

can be proven to be a conservative estimator for the sampling variance of $\bar{Y}$ (e.g., Wolter, 1985, Theorem 2.4.1). On the other hand, there is no way to prove that (3) is conservative under the unaligned scheme. Nevertheless, owing to the comparability of aligned and unaligned schemes in most situations, they should give rise to very similar variances. Thus, (3) should also provide a basically conservative estimator of (2). Finally, note that for the subsequent second-phase estimation of (3), this quantity can be suitably rewritten as

$\frac{S_{Y}^{2}}{N}=\frac{1}{N^{2}} \sum_{i=1}^{N} Y_{i}^{2}-\frac{2}{N^{2}(N-1)} \sum_{h>i=1}^{N} Y_{i} Y_{h}$

\subsection{Second-phase sampling}

In order to reduce survey costs and time, only a portion of points out of the $N$ points selected in the first phase are visited in the second phase to quantify the $Y_{i}$ s. Accordingly, the first-phase estimator $\bar{Y}$ is actually unknown and has to be estimated from a second-phase sample. In order to achieve a two-phase estimator of $T P V$ it is worth noting that, conditional on the set of selected points $\mathrm{P}$, the $Y_{i}$ S constitute a finite population of $N$ values. Hence, (1) may be viewed as the mean of the finite population $Y_{1}, \ldots, Y_{N}$ and as such it may be estimated by means of a sample $\mathrm{Q}$ of $n$ points selected from $\mathrm{P}$ and subsequently visited to achieve the $Y_{i}$ s.
Consider a fixed-size sampling scheme adopted to select a sample $Q$ of $n$ points from P before starting the field work or, alternatively, a random-size scheme adopted for the on-site selection of points during the field work. In both cases, the scheme determines the first-order inclusion probability $\pi_{i}$ and the secondorder inclusion probability $\pi_{i h}$ for $h>i=1, \ldots, N$. Thus, in accordance with Särndal et al. (1992, chapter 9), the doubleexpansion estimator of TPV turns out to be

$\hat{\bar{Y}}=\frac{1}{N} \sum_{i \in Q} \frac{Y_{i}}{\pi_{i}}$

From the properties of the double-expansion estimator (Särndal et al., 1992, Section 9.3), $\hat{Y}$ turns out to be an unbiased estimator of $T P V$, in the sense that

$E_{P \wedge Q}(\hat{\bar{Y}})=T P V$

with variance

$V_{P \wedge Q}(\hat{\bar{Y}})=E_{P}\left\{V_{Q}(\hat{\bar{Y}} \mid P)\right\}+V_{P}(\bar{Y})$

where the operators $E_{P \wedge Q}(\cdot)$ and $V_{P \wedge Q}(\cdot)$ now refer to both firstand second-phase sampling, the operators $E_{Q}(\cdot \mid P)$ and $V_{Q}(\cdot \mid P)$ refer to all the possible second-phase samples $Q$ conditional to the set of points $P$ selected in the first phase, and

$V_{Q}(\hat{\bar{Y}} \mid P)=\frac{1}{N^{2}} \sum_{i=1}^{N} \frac{1-\pi_{i}}{\pi_{i}} Y_{i}^{2}+\frac{2}{N^{2}} \sum_{h>i=1}^{N} \frac{\pi_{i h}-\pi_{i} \pi_{h}}{\pi_{i} \pi_{h}} Y_{i} Y_{h}$

constitutes the sampling variance of $\hat{\bar{Y}}$ conditional to $P$.

As to the estimation of $V_{P \wedge Q}(\hat{\bar{Y}})$, if the design ensures $\pi_{i h}>0$ for any $h>i=1, \ldots, N$, then the first term of (6) can be unbiasedly estimated by the quantity (Särndal et al., 1992, expression 2.8.6)

$\hat{v}^{2}=\frac{1}{N^{2}} \sum_{i \in Q} \frac{1-\pi_{i}}{\pi_{i}^{2}} Y_{i}^{2}+\frac{2}{N^{2}} \sum_{h>i \in Q} \frac{\pi_{i h}-\pi_{i} \pi_{h}}{\pi_{i} \pi_{h}} \frac{Y_{i} Y_{h}}{\pi_{i h}}$

while, in the case of fixed-size designs, the estimator

$\hat{v}^{2}=\frac{1}{N^{2}} \sum_{h>i \in Q} \frac{\pi_{i} \pi_{h}-\pi_{i h}}{\pi_{i h}}\left(\frac{Y_{i}}{\pi_{i}}-\frac{Y_{h}}{\pi_{h}}\right)^{2}$

referred to as the Sen-Yates-Grundy estimator is more commonly used (Särndal et al., 1992, expression 2.8.11). Moreover, according to (4) an unbiased estimator of the second term in (6) is given by

$\frac{\hat{S}_{Y}^{2}}{N}=\frac{1}{N^{2}} \sum_{i \in Q} \frac{Y_{i}^{2}}{\pi_{i}}-\frac{2}{N^{2}(N-1)} \sum_{h>i \in Q} \frac{Y_{i} Y_{h}}{\pi_{i h}}$

Then, it can be proven that $\hat{V}^{2}=\hat{v}^{2}+\hat{S}_{Y}^{2} / N$ is a conservative estimator of (6), i.e., $E_{P \wedge Q}\left(\hat{V}^{2}\right)>V_{P \wedge Q}(\hat{\bar{Y}})$, providing that $S_{Y}^{2} / N$ is a conservative estimator of $V_{P}(\bar{Y})$.

\subsection{Second-phase sampling with auxiliary variables}

When surveys are performed in relatively large areas, all the $N$ first-phase points are usually visited for, at least, a qualitative and informal monitoring of forest attributes. On the other hand, the estimation of $T P V$, requiring fairly cumbersome measurements, is performed only for the $n$ points of the second-phase sample $\mathrm{Q}$.

If Bitterlich sampling is adopted, the Horvitz-Thompson estimates of the total for basal areas

$X_{i}=R \sin ^{2} \frac{\theta}{2}\left\{\left(S_{i}\right)\right\}$

can be readily performed on each first-phase point, since it entails no measurement except for the enumeration of those trees subtending the angle $\theta$. 
As is known in dendrometric studies (e.g., van Laar and Akça, 2007), a strong linear relationship passing through the origin has been proven between the volumes $\left(y_{j}\right)$ and basal areas $\left(b_{j}\right)$ at tree level. Accordingly, a similar relationship is likely to occur between the $X_{i}$ s and the $Y_{i}$ s, in such a way that the $X_{i}$ s can be adopted as auxiliary information to use in the second phase. Quoting again from dendrometric literature, auxiliary information can be taken into account by means of an assisting model of type

$Y_{i}=\beta X_{i}+\varepsilon_{i}, \quad i=1, \ldots, N$

where the $\varepsilon_{i}$ s are assumed to have zero expectations and variances proportional to the $X_{i}$ s. Model (12) is usually referred to as the common ratio model (see e.g., Särndal et al., 1992, section 7.3).

In a similar way, when plot sampling is adopted instead of Bitterlich sampling, an ocular evaluation of the tree volumes within the plots, say $X_{i}$, can be easily performed on each first-phase point and the $X_{i}$ s can serve again as second-phase auxiliary information. Since $X_{i}$ will be 0 when $Y_{i}=0$ (i.e., no trees in the plot) and since evaluation errors should increase in size as the actual volumes increase, a common ratio model could serve to link ocular evaluations and actual volumes (Corona, 2006). Accordingly, it may be reasonable that

$X_{i}=\beta^{*} Y_{i}+\varepsilon_{i}^{*}, \quad i=1, \ldots, N$

where the $\varepsilon_{i}^{*}$ s have zero expectations and variances proportional to the $Y_{i}$ s. Obviously, relation (13) must be reversed to obtain an assisting model similar to (12) with $\beta=1 / \beta^{*}$ and $\varepsilon_{i}=-\varepsilon_{i}^{*} / \beta^{*}$. Note however that the resulting model differs from the genuine ratio model since the $\varepsilon_{i}$ s have 0 expectations but the variances are proportional to the $Y_{i}$ s. However, since $X_{i}$ increase with $Y_{i}$, the variances of the $\varepsilon_{i}$ s may also be considered proportional to the $X_{i}$ s.

From Särndal et al. (1992, example 6.4.2), the common ratio model (12) leads to the ratio estimator

$\hat{\bar{Y}}_{\text {ratio }}=\hat{\beta} \bar{X}$

where $\bar{X}$ denotes the population mean of the $X_{i}$ s, $\hat{\beta}=\hat{\bar{Y}} / \hat{\bar{X}}$, while

$\hat{\bar{X}}=\frac{1}{N} \sum_{i \in Q} \frac{X_{i}}{\pi_{i}}$

denotes the double-expansion estimator for $\bar{X}$. Quoting again from Särndal et al. (1992, example 6.6.1), the ratio estimator is approximately unbiased, in the sense that $E_{Q}\left(\hat{\bar{Y}}_{\text {ratio }} \mid P\right) \approx \bar{Y}$, with approximate variance

$V_{Q}\left(\hat{Y}_{\text {ratio }} \mid P\right) \approx \frac{1}{N^{2}} \sum_{i=1}^{N} \frac{1-\pi_{i}}{\pi_{i}} e_{i}^{2}+\frac{2}{N^{2}} \sum_{h>i=1}^{N} \frac{\pi_{i h}-\pi_{i} \pi_{h}}{\pi_{i} \pi_{h}} e_{i} e_{h}$

where $e_{i}=Y_{i}-\beta X_{i}$.

Accordingly, $\hat{\bar{Y}}_{\text {ratio }}$ turns out to be an approximately unbiased estimator of $T P V$, in the sense that

$E_{P \wedge Q}\left(\hat{\bar{Y}}_{\text {ratio }}\right) \approx T P V$

with an approximate overall variance

$V_{P \wedge Q}\left(\hat{\bar{Y}}_{\text {ratio }}\right) \approx E_{P}\left\{V_{Q}\left(\hat{\bar{Y}}_{\text {ratio }} \mid P\right)\right\}+V_{P}(\bar{Y})$

Finally, as to the estimation of $V_{P \wedge Q}\left(\hat{\bar{Y}}_{\text {ratio }}\right)$, if the design ensures $\pi_{i h}>0$ for any $h>i=1, \ldots, N$, then, as is customary in ratio estimation (Särndal et al., 1992, example 6.6.1), the first term of (16) can be estimated by means of

$\hat{v}_{\text {ratio }}^{2}=\left(\frac{\bar{X}}{\hat{\bar{X}}}\right)^{2} \frac{1}{N^{2}} \sum_{i \in Q} \frac{1-\pi_{i}}{\pi_{i}^{2}} \hat{e}_{i}^{2}+2\left(\frac{\bar{X}}{\hat{\bar{X}}}\right)^{2} \frac{1}{N^{2}} \sum_{h>i \in Q} \frac{\pi_{i h}-\pi_{i} \pi_{h}}{\pi_{i} \pi_{h}} \frac{\hat{e}_{i} \hat{e}_{h}}{\pi_{i h}}$ or, in the case of fixed-size designs, by means of

$\hat{v}_{\text {ratio }}^{2}=\left(\frac{\bar{X}}{\hat{\bar{X}}}\right)^{2} \frac{1}{N^{2}} \sum_{h>i \in Q} \frac{\pi_{i} \pi_{h}-\pi_{i h}}{\pi_{i h}}\left(\frac{\hat{e}_{i}}{\pi_{i}}-\frac{\hat{e}_{h}}{\pi_{h}}\right)^{2}$

where $\hat{e}_{i}=Y_{i}-\hat{\beta} X_{i}$.

Since the second-phase estimator of the second term in (16) is identical to (10), the quantity $\hat{V}_{\text {ratio }}^{2}=\hat{v}_{\text {ratio }}^{2}+\hat{S}_{Y}^{2} / N$ is again conservative when $S_{Y}^{2} / N$ is a conservative estimator of $V_{P}(\bar{Y})$.

\subsection{Second-phase schemes}

Three schemes for the selection of second-phase samples are considered: simple random sampling without replacement (SRSWOR), stratified sampling (STRS) and 3-P sampling (3PS). The first two fixed-size schemes involve the selection of $Q$ before starting the field work while 3PS is a random-size scheme and involves on-site selections during the field work.

In the case of SRSWOR, $n$ points are randomly selected without replacement from P; the first- and second-order inclusion probabilities under SRSWOR are

$\pi_{i}=\frac{n}{N}$

and

$\pi_{i h}=\frac{n(n-1)}{N(N-1)}$

(see e.g., Särndal et al., 1992, Example 2.4.1). Alternatively, in order to achieve a more even distribution of second-phase points over the study area, the $N$ first-phase points may be partitioned into $L$ spatial strata of equal size. Subsequently, $n / L$ points are selected within each stratum by means of SRSWOR. In this case the firstorder inclusion probabilities are the same as with SRSWOR, while the second-order probabilities are

$\pi_{i h}=\frac{n(n-L)}{N(N-L)}$

when points $i$ and $h$ are in the same stratum and

$\pi_{i h}=\frac{n^{2}}{N^{2}}$

when points $i$ and $h$ are in different strata.

To perform 3PS, the largest possible $X_{i}$, say $X_{\mathrm{MAX}}$ is presumed and a constant $C>X_{\operatorname{MAX}}$ is chosen. Then, during the field work, following the recording of each auxiliary value $X_{i}$, the point is selected with probability

$\pi_{i}=\frac{X_{i}}{C}$

In 3PS sample size $n$ is a random variable with expectation

$E_{P \wedge Q}(n)=E_{P}\left\{E_{Q}(n \mid P)\right\}=E_{P}\left\{\frac{N \bar{X}}{C}\right\}$

Moreover, since the selection of points is performed independently, the second-order inclusion probabilities are

$\pi_{i h}=\pi_{i} \pi_{h}=\frac{X_{i} X_{h}}{C^{2}}$

For more details on 3PS, see e.g., De Vries (1986, chapter 15) and Gregoire and Valentine (2008, Section 11.7).

First- and second-order inclusion probabilities of these schemes are inserted in the expressions (5), (8), (9) and (10) to give the double-expansion estimator $\hat{Y}$ and its variance estimators or into expressions (14), (17) and (18) to obtain the ratio estimator $\hat{\bar{Y}}_{\text {ratio }}$ and its variance estimators. 


\section{A Monte Carlo experiment}

\subsection{Materials}

The performance of double-expansion and ratio estimators under SRSWOR, STRS and 3PS was checked by means of simulating sampling in a real population. The study area $\mathcal{A}$ was a forest compartment of size $A=11.934$ ha located within the beech (Fagus sylvatica L.) forest of Monte Cimino (Central Italy). For a detailed description of the study area, see Lamonaca et al. (2008) (Fig. 1).

A complete field enumeration gave tree volumes $\left(y_{j}\right)$ and basal areas $\left(b_{j}\right)$ for the whole population of 2734 trees and $T P V=9049.313 \mathrm{~m}^{3}$. Fig. 2 plots tree basal areas against their volumes. An investigation performed by a MSc Student on the beech forest including the study area gave actual values $\left(Y_{i}\right)$ and ocular evaluations $\left(X_{i}\right)$ of stand volumes in 37 plots centered on a systematic grid of points; Fig. 3 plots ocular evaluations against

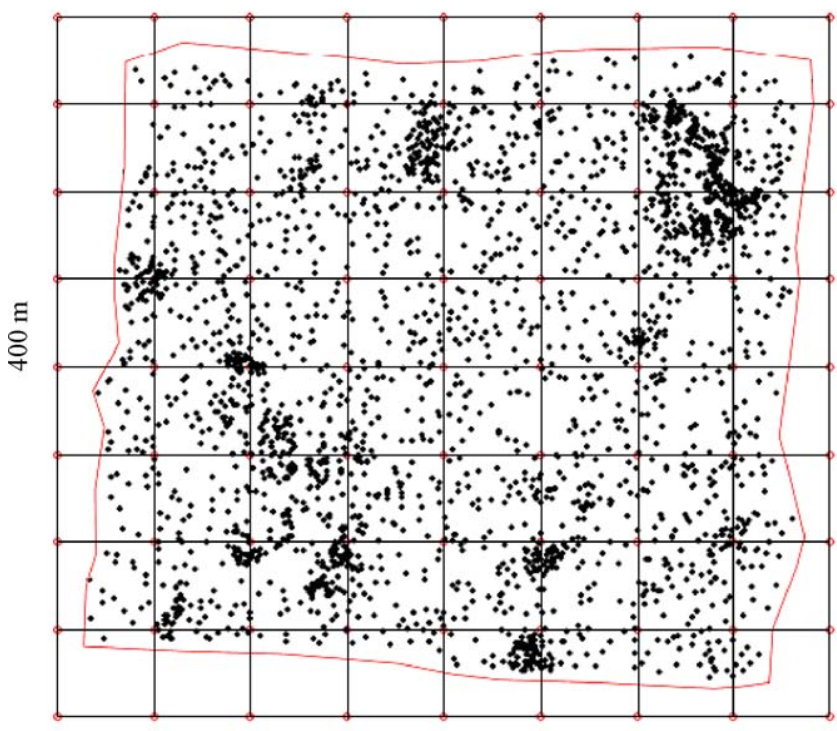

$400 \mathrm{~m}$

Fig. 1. Graphical representation of the study area and covering region. Marked points within the study area represent tree locations.

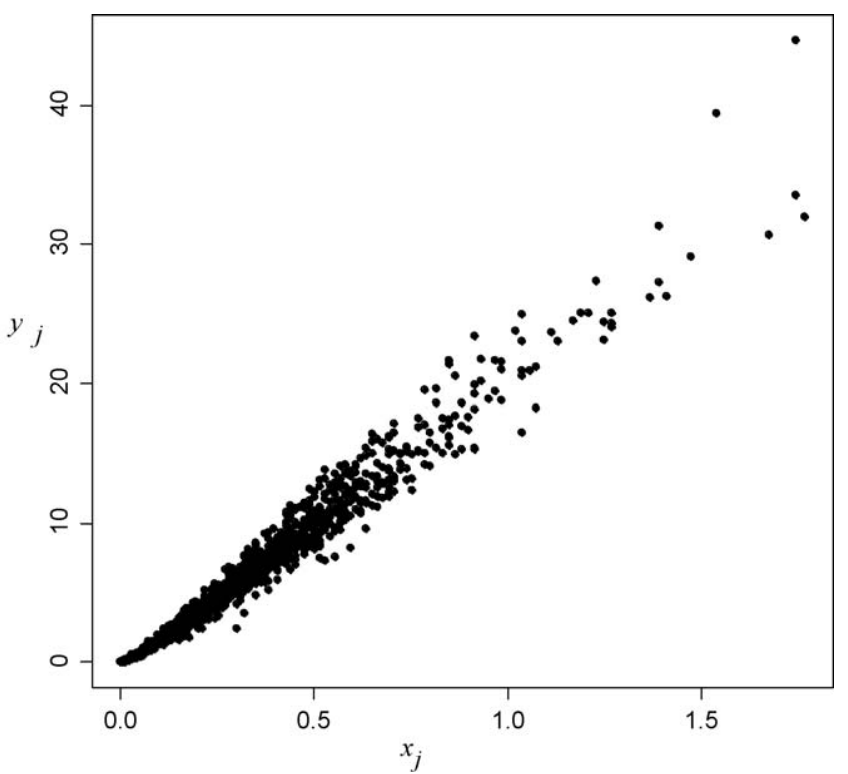

Fig. 2. Scatterplot of basal areas $\left(b_{j}\right)$ against volumes $\left(y_{j}\right)$ for the 2734 trees within the study area.

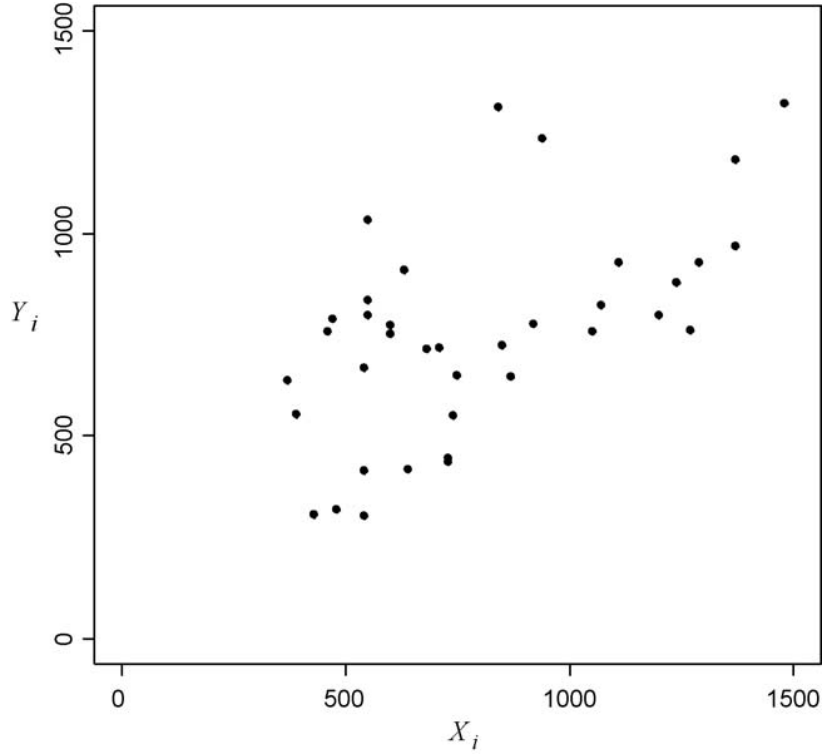

Fig. 3. Scatterplot of ocular evaluations $\left(X_{i}\right)$ against true volumes $\left(Y_{i}\right)$ in 37 plots centered on a systematic grid of points superimposed onto the beech forest including the study area.

true volumes. Both the scatterplots support the common ratio model in (12).

\subsection{Experiment setting}

To perform unaligned systematic sampling, the study area was covered by a quadrat region $\mathcal{R}$ of size $\mathcal{R}=16$ ha (side $400 \mathrm{~m}$ ). To simulate an intensive inventory, $\mathcal{R}$ was partitioned into $N=64$ quadrats of size 0.25 ha (side $50 \mathrm{~m}$ ) (see Fig. 1) and 10,000 firstphase samples were randomly generated by means of unaligned systematic sampling. Accordingly, each simulated first-phase sample $\mathrm{P}$ had $N=64$ points in which one point was randomly selected in a quadrat (e.g., the first quadrat) with the other points systematically repeated in the $N-1$ remaining quadrats.

For each point $\mathbf{u}_{i} \in \mathrm{P}$ the value of the auxiliary variable was determined. In Bitterlich sampling, $X_{i}$ is the basal area estimate obtained from the trees subtending an angle less than $\theta=0.02828$ radiants $\left(=1.6206^{\circ}\right)$ onto $\mathbf{u}_{i}$. Such an angle corresponds to a BAF of 2 , which is commonly used in forest inventories. For this BAF, the basal area estimate in (11) expressed in $\mathrm{m}^{2}$ reduces to $X_{i}=2 \times R \times \#\left(\mathrm{~S}_{i}\right)$. In plot sampling, the auxiliary variable $X_{i}$ is the ocular evaluation of total volume within the plot of radius $15 \mathrm{~m}$ (size $a=706.858 \mathrm{~m}^{2}$ ) centred at $\mathbf{u}_{i}$. Evaluations were generated on the basis of the following assumptions: (a) the evaluations were fixed for a given $\mathbf{u} \in \mathcal{R}$; (b) also, similar evaluations were expected for points $\mathbf{u}_{1}, \mathbf{u}_{2}$ in close proximity; (c) evaluation errors increased in size as the actual volume increased. Accordingly, the evaluation at a point $\mathbf{u} \in \mathcal{R}$ was assumed to be a deterministic continuous function of $\mathbf{u}$ of type

$X(\mathbf{u})=k Y(\mathbf{u})+\beta Y(\mathbf{u}) \varphi(\mathbf{u})$

where $k$ and $\beta$ represented positive parameters, $Y(\mathbf{u})$ denoted the actual predicted volume within the plot of radius $15 \mathrm{~m}$ centred at $\mathbf{u}$,

$\varphi(\mathbf{u})=\frac{a\left(v_{1}\right)+a\left(v_{2}\right)+a\left(v_{1} v_{2}\right)}{6}$

represented a fluctuation in the range $-1,1, v_{1}$ and $v_{2}$ constitute the two coordinates of $\mathbf{u}$ rescaled as to vary between 0 and 1 and $a(v)=\sin \left(-\frac{\pi}{2}+v \pi\right)+\cos (v \pi)$ 
Practically speaking, the ocular evaluation of volume was assumed to be a linear function of the actual volume in the plot plus a positive or negative perturbation increasing in size with the actual volume. In order to allow situations where a forest technician tends to over-evaluate or under-evaluate the actual volume (Corona, 2006), $k$ was set equal to 1.2 and 0.8 while $\beta$ was set to 0.8 throughout.

After $X_{i}$ s were determined for the $N=64$ first-phase points, a second-phase sample $\mathrm{Q}$ of size $n=8,16$ was selected from $\mathrm{P}$ by means of SRSWOR, STRS with $L=4$ spatial strata determined by the four quadrats of size 4 ha (side $200 \mathrm{~m}$ ) partitioning $\mathcal{R}$ and 3PS. The value of $C$ in 3PS was chosen to give the same average sample size as in SRSWOR and STRS.

\subsection{Outputs}

For each simulated second-phase sample $\mathrm{Q}$ the following statistics were computed: double-expansion estimate $\hat{\bar{Y}}$, variance estimate $\hat{V}^{2}$, relative standard error estimate $R \hat{S} E=\hat{V} / \hat{Y}, 0.95$ confidence interval $\hat{\bar{Y}} \pm 1.96 \hat{V}$; and its width $W=1.96 \hat{V}$, ratio

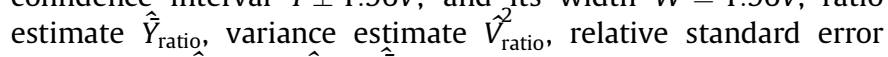
estimate $R \hat{S} E_{\text {ratio }}=\hat{V}_{\text {ratio }} / \hat{\bar{Y}}_{\text {ratio }}, \quad 0.95$ confidence interval $\hat{\bar{Y}}_{\text {ratio }} \pm 1.96 \hat{V}_{\text {ratio }} ;$ and its width $W_{\text {ratio }}=1.96 \hat{V}_{\text {ratio }}$.

Monte Carlo distributions of the above statistics were used to derive the following expectations: $E_{P \wedge Q}(\hat{\bar{Y}}), M S E_{P \wedge Q}(\hat{\bar{Y}})=E_{P \wedge Q}$ $\left\{(\hat{\bar{Y}}-T P V)^{2}\right\}, E_{P \wedge Q}(R \hat{S} E), E_{P \wedge Q}(W), E_{P \wedge Q}\left(\hat{\bar{Y}}_{\text {ratio }}\right), M S E_{P \wedge Q}\left(\hat{\bar{Y}}_{\text {ratio }}\right)=$ $E_{P \wedge Q}\left\{\left(\hat{\bar{Y}}_{\text {ratio }}-T P V\right)^{2}\right\}, E_{P \wedge Q}\left(R \hat{S} E_{\text {ratio }}\right), E_{P \wedge Q}\left(W_{\text {ratio }}\right)$.

In turn, the above expectations allowed for the computation of: relative bias $(\mathrm{RB}),\left\{E_{P \wedge Q}(\hat{\bar{Y}})-T P V\right\} / T P V$ and $\left\{E_{P \wedge Q}\left(\hat{\bar{Y}}_{\text {ratio }}\right)-\right.$ $T P V\} / T P V$, relative root mean squared error (RRMSE), $\sqrt{M S E_{P \wedge Q}(\hat{\bar{Y}})} / T P V$ and $\sqrt{M S E_{P \wedge Q}\left(\hat{\bar{Y}}_{\text {ratio }}\right)} / T P V$, expected relative standard error estimate (ERSEE), $E_{P \wedge Q}(R \hat{S E})$ and $E_{P \wedge Q}\left(R \hat{S} E_{\text {ratio }}\right)$ and expected confidence interval relative width (ECIRW), $E_{P \wedge Q}(W) / T P W$ and $E_{P \wedge Q}\left(W_{\text {ratio }}\right) / T P W$. Finally the actual coverage (AC) of the confidence intervals was determined as the percentage of times the intervals contained the true value of TPV.

\subsection{Results}

Tables 1-3 report the percent values of RB, RRMSE, ERSEE, AC and ECIRW for both Bitterlich and plot sampling (involving ocular over- and under-evaluations) performed on $N=64$ first-phase points or plots, with $n=8,16$ points selected in the second phase by means of SRSWOR. The results obtained using STRS and 3PS are not reported here for brevity, since they are completely equivalent to those achieved with SRSWOR.

There is negligible bias in results with double-expansion and ratio estimators. Absolute values of RB are always less than $0.3 \%$ and, in most cases, less than $0.1 \%$. In all settings the ratio estimator shows important reduction of RRMESs when compared to the

Table 1

Performance of double-expansion and ratio estimators in the case of Bitterlich sampling performed on $N=64$ first-phase sampling points $(\mathrm{BAF}=2)$. Second-phase scheme: SRSWOR.

\begin{tabular}{lllllr}
\hline Sample size & 8 & & & 16 & \\
\cline { 2 - 3 } \cline { 6 - 6 } \cline { 5 - 6 } & $\begin{array}{l}\text { Double-expansion } \\
\text { estimator }\end{array}$ & $\begin{array}{l}\text { Ratio } \\
\text { estimator }\end{array}$ & & $\begin{array}{l}\text { Double-expansion } \\
\text { estimator }\end{array}$ & $\begin{array}{l}\text { Ratio } \\
\text { estimator }\end{array}$ \\
\hline RB (\%) & $-0.23 \%$ & $-0.05 \%$ & & $-0.07 \%$ & $-0.11 \%$ \\
RRMSE (\%) & $18.05 \%$ & $3.90 \%$ & & $11.93 \%$ & $2.98 \%$ \\
ERSEE (\%) & $19.27 \%$ & $7.53 \%$ & & $13.59 \%$ & $7.13 \%$ \\
AC (\%) & $91.78 \%$ & $99.81 \%$ & & $95.72 \%$ & $100.00 \%$ \\
ECIRW (\%) & $73.44 \%$ & $28.90 \%$ & & $52.73 \%$ & $27.68 \%$ \\
\hline
\end{tabular}

Table 2

Performance of double-expansion and ratio estimators in the case of plot sampling with ocular evaluations performed on $\mathrm{N}=64$ first-phase plots of radius $15 \mathrm{~m}$. Ocular evaluations are determined by means of Eq. (19) with $k=1.2$ (overevaluations). Second-phase scheme: SRSWOR.

\begin{tabular}{llllll}
\hline Sample size & 8 & & & 16 & \\
\cline { 2 - 3 } \cline { 6 - 6 } & $\begin{array}{l}\text { Double-expansion } \\
\text { estimator }\end{array}$ & $\begin{array}{l}\text { Ratio } \\
\text { estimator }\end{array}$ & & $\begin{array}{l}\text { Double-expansion } \\
\text { estimator }\end{array}$ & $\begin{array}{l}\text { Ratio } \\
\text { estimator }\end{array}$ \\
\hline RB (\%) & $-0.18 \%$ & $0.23 \%$ & & $-0.00 \%$ & $-0.01 \%$ \\
RRMSE (\%) & $23.69 \%$ & $8.30 \%$ & & $16.30 \%$ & $7.44 \%$ \\
ERSEE (\%) & $24.24 \%$ & $9.53 \%$ & & $17.09 \%$ & $9.04 \%$ \\
AC (\%) & $91.00 \%$ & $95.61 \%$ & & $93.78 \%$ & $97.77 \%$ \\
ECIRW (\%) & $92.42 \%$ & $36.72 \%$ & & $66.22 \%$ & $35.07 \%$ \\
\hline
\end{tabular}

Table 3

Performance of double-expansion and ratio estimators in the case of plot sampling with ocular evaluations performed on $\mathrm{N}=64$ first-phase plots of radius $15 \mathrm{~m}$. Ocular evaluations are determined by means of Eq. (19) with $k=0.8$ (underevaluations). Second-phase scheme: SRSWOR.

\begin{tabular}{llllll}
\hline Sample size & 8 & & & 16 & \\
\cline { 2 - 3 } & $\begin{array}{l}\text { Double-expansion } \\
\text { estimator }\end{array}$ & $\begin{array}{l}\text { Ratio } \\
\text { estimator }\end{array}$ & & $\begin{array}{l}\text { Double-expansion } \\
\text { estimator }\end{array}$ & $\begin{array}{l}\text { Ratio } \\
\text { estimator }\end{array}$ \\
\hline RB (\%) & $-0.18 \%$ & $0.53 \%$ & & $-0.00 \%$ & $0.12 \%$ \\
RRMSE (\%) & $23.69 \%$ & $9.86 \%$ & & $16.30 \%$ & $8.20 \%$ \\
ERSEE (\%) & $24.24 \%$ & $10.57 \%$ & & $17.09 \%$ & $9.60 \%$ \\
AC (\%) & $91.00 \%$ & $94.09 \%$ & & $93.78 \%$ & $97.23 \%$ \\
ECIRW (\%) & $92.42 \%$ & $40.88 \%$ & & $66.22 \%$ & $37.30 \%$ \\
\hline
\end{tabular}

double-expansion estimator. In contrast to the double-expansion estimator, the ratio estimator always gave conservative estimates of RRMSE and confidence intervals with actual coverage which are above the nominal level of 95\%. Moreover, the confidence intervals constructed around ratio estimates are much shorter than intervals obtained via double-expansion estimates.

\section{Discussion and conclusion}

Inventory by compartments at $10-15$ year intervals is still very common method for conducting surveys in support of forest management planning in Europe. In some countries, large forest companies and forest enterprises appear to prefer continuous inventory (Koivuniemi and Korhonen, 2006). The basic ideas behind inventory by compartments originated in Central Europe long before probabilistic sampling theory was developed for this type of estimation problem. More recently many authors (e.g., Laasasenaho and Paivinen, 1986; Stähl, 1992; Haara, 2005; Kinnunen et al., 2007) have acknowledged that errors in compartment-level estimates can be reduced if supplemented with probability sampling.

The results from the Monte Carlo experiment highlight the importance of using probabilistic sampling in the estimation of growing stock volume at the compartment level. Indeed, bias has proved to be negligible for both double-expansion and ratio estimators as well as under Bitterlich and plot sampling and for any second-phase scheme. This finding is of particular interest with respect to conventional inventories by compartments, in which discrepancies over $10 \%$ are frequently observed between single guess/ocular assessments and census results (e.g., Nersten and Næsset, 1992; Stähl, 1992; Kinnunen et al., 2007). Our results have demonstrated how easy and fast auxiliary data can improve the efficiency of probabilistic volume sampling.

Basal area estimates (Bitterlich sampling) provides more accurate estimation than ocular evaluations (plot sampling). Notwithstanding, ocular evaluation remains an option owing to 
its great practical feasibility (e.g., Rondeaux et al., 1998; Bousson et al., 2002; Kangas et al., 2004; Lejeune et al., 2005). Ocular evaluations may also work reasonable well with less than fully experienced technicians as long as they are consistent in their calls. Indeed, ocular evaluations are only adopted to calibrate the second-phase estimate, so that under- or over-evaluations do not greatly affect the bias of the final ratio estimate. Instead, evaluation errors do more harm to the accuracy of the ratio estimator than the use of objective information such as basal area estimates.

A critical aspect of the proposed strategies is the need for rigorous geo-location of the sampling units. Fortunately, it has been greatly facilitated over the last decades. A rigorous location of points is especially critical for the Bitterlich method; even nearby point locations may produce substantially different estimates. Ocular plot sampling is more robust against location errors; a certain tolerance may be admitted in positioning the sampling units.

In relatively large compartments containing, for examples, a mix of different species, silvicultural treatments or age structures, a stratification may be necessary to improve the efficiency of the sampling design. In that case reference should be made to the proper inclusion probabilities of second-phase units (see Section 2.4).

With efficiently updated inventory by compartment, strategic and tactical planning and decisions can be based on the timely knowledge of local forest resources. Operative planning and subsequent activities may become more cost-efficient. On the basis of Italian experience (Corona and Ferrara, 1989; Corona, 2006), the total time required by a two-technician team to perform the two-phase inventory described in the simulation is about a fourth of the time needed to calliper all the trees and measure a sample of heights or about twice the time necessary to perform first-phase ocular or relascope assessment. Two-phase inventories of local forest attributes provide a compromise between very intensive field assessments in which all the first-phase points are considered and reliance on auxiliary data correlated with the variable of interest but not sufficient for the specific inventory task. Auxiliary data such as the basal area or ocular evaluations can indeed be adopted to assist and improve the design-based estimation of forest volumes but they are not sufficient to provide a direct estimation, unless models relating the auxiliary data to volumes are introduced. In these cases the inference is of a modelbased nature and the resulting estimators are strictly dependent on model validity.

From a practical point of view, a two-phase sampling is attractive when (i) the unit cost of acquiring first-phase data is significantly lower than that of second-phase data; (ii) the auxiliary information collected in the first phase is well correlated with the estimates performed at second-phase points. Both these requirements are met in the present study. As such, the strategies proposed in the paper seem to merit further investigation to explore their effectiveness under various compositional, structural and silvicultural conditions.

\section{Acknowledgements}

The work was partially carried out under the "Carboltaly" project (DISAFRI research unit) funded by the FISR program of the
Italian Ministry of University and Research, and partially under the COFIN PRIN 2007 project "Innovative methods for the identification, characterization and management of old-growth forests in the Mediterranean environment" (DISAFRI research unit) funded by the same Ministry.

\section{References}

Barabesi, L., 2003. A Monte Carlo integration approach to Horvitz-Thompson estimation in replicated environmental designs. Metron 61, 355-374.

Bousson, E., Lejeune, P., Rondeux, J., 2002. L'inventaire par évaluation visuelle, une nouvelle méthode adaptée a la description de peuplements hétérogènes. Forêt Wallonne 58, 6-12.

Corona, P., 2006. Sperimentazione di procedure probabilistiche innovative per la cubatura della massa legnosa a fini assestamentali. Testing probabilistic techniques of timber volume estimation for forest management planning. Forest@ 2, 267-274 (in Italian with English summary).

Corona, P., Ferrara, A., 1989. La combinazione ottimale tra numero e superficie unitaria delle aree campione nei rilievi dendrometrici ordinary. The optimum combination between number and size of sampling plots in forest mensuration. Annali Accademia Italiana di Scienze Forestali 38, 109-132 (in Italian with English summary).

De Vries, P.G., 1986. Sampling Theory for Forest Inventories. Springer-Verlag, New York.

EPA, 2002. Guidance for Choosing a Sampling Design for Environmental Data Collection. EPA QA/G-5S. US Environmental Protection Agency, Washington, DC.

FAO, 2004. Global forest assessment update 2005. Terms and definitions. FRA Programme, Working Paper 83/E. Rome.

Gregoire, T.G., 1998. Design-based and model-based inference in survey sampling: appreciating the difference. Canadian Journal of Forest Research 28, 1429-1447.

Gregoire, T.G., Valentine, H.T., 2008. Sampling Strategies for Natural Resources and the Environment. Chapmann \& Hall, New York.

Haara, H., 2005. The uncertainty of forest management planning data in Finnish non-industrial private forestry. Dissertationes Forestales 8. Joensuun Yliopistopaino, Joensuu.

Kangas, A., Heikkinen, E., Maltamo, M., 2004. Accuracy of partially visually assessed stand characteristics: a case study of Finnish forest inventory by compartments. Canadian Journal of Forest Research 34, 916-930.

Kinnunen, J., Maltamo, M., Päivinen, R., 2007. Standing volume estimates of forests in Russia: how accurate is the published data? Forestry 80, 53-64.

Köhl, M., Magnussen, S., Marchetti, S., 2006. Sampling Methods, Remote Sensing and GIS Multiresource Forest Inventory. Tropical Forestry Series. Springer-Verlag, Berlin-Heidelberg.

Koivuniemi, J., Korhonen, K.T., 2006. Inventory by compartments. In: Kangas, A., Maltamo, M. (Eds.), Forest Inventory. Methodology and Applications. Springer, Dordrecht, pp. 271-278.

Laasasenaho, J., Paivinen, R., 1986. Kuvioittaisen arvioinnin tarkistamisesta. On the checking of inventory by stands. Folia Forestalia, 664 (in Finnish with English summary).

Lamonaca, A., Corona, P., Barbati, A., 2008. Exploring forest structural complexity by multi-scale segmentation of VHR imagery. Remote Sensing of Environment 112 2839-2849.

Lejeune, P., Hébert, J., Bousson, E., Verrue, V., Rondeux, J., 2005. L'inventaire par évaluation visuelle de grosseurs d'arbres, une alternative pertinente aux inventaires forestiers complets. Annals of Forest Sciences 62, 343-349.

Nersten, S., Næsset, E., 1992. Nøyaktighet av bestandtaksering med relaskop. Accuracy of standwise relascope survey. Meddelelser fra Skogforsk 45, 1-22 (in Norwegian with English summary).

Rondeaux, J., Hebert, J., Marchal, D., 1998. Les inventaires forestier de gestion: quels objectifs et quelles méthodes? Journal Forestier Suisse 149, 463-473.

Särndal, C.E., Swensson, B., Wretman, J., 1992. Model Assisted Survey Sampling. Springer-Verlag, New York.

Schreuder, H.T., Gregoire, T.G., Wood, G.B., 1993. Sampling Methods for Multiresource Forest Inventory. Wiley, New York.

Stähl, G., 1992. En studie av kvalitet i skogliga avdelningsdata som insamlats med subjektiva inventerigsmetoder. A study on the quality of standwise forest data acquired by subjective inventory methods. Swedish University of Agricultural Sciences. Department of Biometry and Forest Management, Rapport 24 (in Swedish with English summary).

van Laar, A., Akça, A., 2007. Forest Mensuration. Springer, Dordrecht.

Wolter, K.M., 1985. Introduction to Variance Estimation. Springer-Verlag, New York. 